\title{
After shrinkage apoptotic cells expose internal membrane-derived epitopes on their plasma membranes
}

\author{
S Franz ${ }^{1}$, K Herrmann ${ }^{2}$, B Führnrohr ${ }^{4}$, A Sheriff', B Frey ${ }^{3}$, US GaipI ${ }^{1,5}$, RE Voll ${ }^{4}$, JR Kalden ${ }^{1}$, H-M Jäck ${ }^{2}$ and M Herrmann ${ }^{\star, 1}$
}

Apoptosis and phagocytosis of apoptotic cells are crucial processes. At best the phagocytic machinery detects and swallows all apoptotic cells in a way that progression to secondary necrosis is avoided. Otherwise, inflammation and autoimmune diseases may occur. Most apoptotic cells are phagocytosed instantaneously in a silent fashion; however, some dying cells escape their clearance. If the cells are not cleared early, they lose membranes due to extensive shedding of membrane surrounded vesicles (blebbing) and shrink. It is unclear how apoptotic cells compensate their massive loss of plasma membrane. Here, we demonstrate that endoplasmic reticulum- (ER) resident proteins (calnexin, the KDEL receptor and a dysfunctional immunoglobulin heavy chain) were exposed at the surfaces of shrunken late apoptotic cells. Additionally, these cells showed an increased binding of lectins, which recognize sugar structures predominantly found as moieties of incompletely processed proteins in ER and Golgi. In addition the ER resident lipophilic ER-Tracker ${ }^{\mathrm{TM}}$ Blue-White DPX, and internal GM1 were observed to translocate to the cell surfaces during late apoptosis. We conclude that during blebbing of apoptotic cells the surface membrane loss is substituted by immature membranes from internal stores. This mechanism explains the simultaneous appearance of preformed recognition structures for several adaptor proteins known to be involved in clearance of dead cells.

Cell Death and Differentiation (2007) 14, 733-742. doi:10.1038/sj.cdd.4402066; published online 15 December 2006

Generally, apoptosis is a cellular suicide executed in a programmed fashion. There are some unifying features found in most cells undergoing apoptosis. In the early phase, cells expose phosphatidylserine (PS) ${ }^{1}$ and get opsonized by various PS ligands (milk fat globule EGF/factor VIII (MFGE8), $\beta 2$ gp1, growth arrest-specific gene 6 (Gas-6)) which mediate uptake by phagocytes via integrins, Mer kinase and other scavenger receptors. ${ }^{2}$ This clearance process suppresses inflammatory and immune reactions.

If apoptotic cells escape immediate clearance, they start blebbing and concomitantly reduce their volume. The shrunken cells then bind additional ligands like C1q, C-reactive protein (CRP), the long pentraxin (PTX-3), and the surfactant protein $A$ and $D$ (SP-A and $-D){ }^{2}$ These adaptor proteins mediate a second wave of clearance for those cells that had escaped the early PS-dependent phagocytosis and help avoid the entry of apoptotic cells into secondary necrosis. The apoptotic cell bound opsonins are then recognized via phagocyte receptors like Fc $\gamma$ RIIA, C1q receptor, CR1, CD91 and calreticulin. ${ }^{2}$ Cells dying after severe impact and apoptotic cells that are not cleared timely undergo primary and secondary necrosis, respectively. In contrast to apoptosis, necrosis, especially primary necrosis is pro-inflammatory and consecutively immune stimulatory. ${ }^{3}$

In early apoptosis, caspases and other proteases destroy the cytoskeleton step by step. ${ }^{4,5}$ This process leaves the plasma membrane without the underlying net of actin, focal adhesion proteins, ERM (ezrin/radixin/moesin) molecules, plectin, intermediate filaments, and microtubules. Without the support of the cytoskeleton the plasma membrane loses its rigidity. Two effects are indicative for this early apoptotic process where the cytoskeletal anchors are degraded. The plasma membrane gets acid labile ${ }^{6}$ and its fluidity increases significantly as can be observed by increases in the mobility of $\mathrm{PS}^{7}$ Additionally, the retransport of PS from the outer to the inner leaflet of the plasma membrane declines. The increase of the PS exposure on apoptotic cells can be monitored employing annexin $\mathrm{V}$ $(\mathrm{AxV})$. Besides the massive disturbance of plasma membrane morphology, the plasma membrane of apoptotic cells is known to remain ion selective for a long time (propidium iodide (PI) negative).

\footnotetext{
${ }^{1}$ Institute of Clinical Immunology and Rheumatology, Department of Internal Medicine 3, University of Erlangen-Nürnberg, Erlangen, Germany; ${ }^{2}$ Division of Molecular Immunology, Department of Internal Medicine 3, Nikolaus-Fiebiger-Center of Molecular Medicine, University of Erlangen-Nürnberg, Erlangen, Germany; ${ }^{3}$ Department of Radiooncology, University of Erlangen-Nürnberg, Erlangen, Germany; ${ }^{4}$ IZKF Research Group 2, Nikolaus-Fiebiger-Center of Molecular Medicine, University of Erlangen-Nürnberg, Erlangen, Germany and ${ }^{5}$ Department of Experimental Medicine I, Nikolaus-Fiebiger-Center of Molecular Medicine, Friedrich-Alexander-University of Erlangen-Nürnberg, Erlangen, Germany

${ }^{*}$ Corresponding author: M Herrmann, Institute for Clinical Immunology, Department of Internal Medicine III, Friedrich-Alexander University of Erlangen-Nürnberg, Krankenhausstr. 12, 91054 Erlangen, Germany. Tel: +49-9131-85-36345; Fax: + 49-9131-85-35776; E-mail: Martin.Herrmann@med3.imed.uni-erlangen.de Keywords: late apoptosis; cell shrinkage; blebbing; ER; glycosylation

Abbreviations: AxV, annexin V; CRP, C-reactive protein; CT-B, cholera toxin subunit B; ER, endoplasmic reticulum; FCS, foetal calf serum; FSc, forward scatter; Gas6, growth arrest-specific gene 6; GSL II, Griffonia simplificolia lectin II; HC, heavy chain; MFG-E8, milk fat globule EGF/factor VIII; MAL I, Maackia amurensis lectin; NPn, Narcissus pseudonarcissus lectin; PBL, peripheral blood lymphocytes; PBMC, peripheral blood mononuclear cells; PI, propidium iodide; PS, phosphatidylserine; PTX-3, long pentraxin; SLE, systemic lupus erythematosus; SP-A, surfactant protein A; SP-D, surfactant protein D; SSc, side scatter; UEA I, Ulex europaeus agglutinin I Received 30.3.06; revised 04.9.06; accepted 05.10.06; Edited by M Piacentini; published online 15.12.06
} 
Meanwhile, characteristic cytomorphological changes like cytoplasm condensation, chromatin aggregation, and fragmentation of the nucleus can be detected. Later on, apoptotic cells lose volume due to cytoplasm 'boiling' and plasma membrane 'blebbing' and form apoptotic bodies that initially remain surrounded by membranes. ${ }^{8}$

The volume reduction of the shrunken cell (third-order potency) cannot completely compensate for its membrane loss (second-order potency). Therefore, the plasma membrane has to be substituted from internal sources. Former observations suggested that the endoplasmic reticulum (ER) is a candidate for substituting the membrane loss. ${ }^{9}$ However, molecular data supporting this notion are still missing. Recently, it has been demonstrated that the ER is punctually associated with the plasma membrane ${ }^{10}$ and that it substitutes the loss of plasma membrane during the process of particle ingestion. ${ }^{11}$ In recent publications, we and others showed that cells undergoing apoptosis change the glycosylation status of their membranes. ${ }^{12,13}$ The sugar structures that we have detected on late apoptotic cells are usually found as terminal sugar residues of incompletely processed proteins, which have not yet fully passed the ER and Golgi compartments of the cells.

Here, we demonstrate that after shrinkage apoptotic cells show an increased exposure of ER-resident lipids, proteins and immature glycoproteins. We conclude that after membrane blebbing from apoptotic cells, the loss of plasma membrane is directly substituted by internal membranes, at least partially derived from ER. We speculate that immature proteins are passively translocated to the cell surface during the process of shrinkage of late apoptotic cells.

\section{Results}

Lectins recognizing epitopes from immature glycoproteins increase their binding to shrunken, late apoptotic cells. Cell death is characterized by morphological changes. At a certain stage apoptotic cells lose volume caused by the release of membranous surface vesicles (blebs). The flow cytofluorometer routinely measures two basic morphologic attributes of a cell. The cell 'size' and 'granularity', which are reflected by the forward (FSc) and the side scatter (SSc) values, respectively. Figure 1a shows the morphologies of viable Jurkat cells and neutrophils (wo, $0 \mathrm{~h}$ ). Apoptosis-related morphological changes can be seen in cultures of ageing neutrophils (Figure 1a, neutrophils, 23 h) or UV-B irradiated Jurkat cells (Figure 1a, Jurkat, UV-B $8 \mathrm{~h}$ ). In both cases cell populations with lowered FSc values (populations 4) emerge reflecting apoptotic/secondary necrotic cells with a reduced cell volume. Binding of $\mathrm{AxV}$ and exclusion of $\mathrm{PI}$, or penetration of $\mathrm{PI}$ confirmed the apoptotic or secondary necrotic status of these cells, respectively (Figure $1 \mathrm{~b}$, populations 4). Some AxV-binding cells were also detected in the full size cells (Figure 1a and b, populations 2 and 3), reflecting early apoptosis. Figure 1c shows the increased binding to apoptotic cells of the mannose-specific lectin from Narcissus pseudonarcissus (NPn). A similar increase of the binding to apoptotic lymphocytes ${ }^{13}$ and also to apoptotic granulocytes (not shown) was also to be observed with the lectins derived from Griffonia simplificolia (Griffonia simplificolia lectin II (GSL II)), and Ulex europaeus (UEA I), recognizing $\mathrm{N}$-acetylglucosamine, and fucose, respectively.

Before shrinkage apoptotic cells lose sialic acid epitopes characterizing mature glycoproteins as detected by binding of Maackia amurensis lectin I. We used the lectin Maackia amurensis lectin I (MAL I) to detect changes of the glycosylation status of ageing neutrophils, and irradiated Jurkat cells (Figure 1c). The experiment showed that the amount of accessible sugar ligands for MAL I decreased from viable to apoptotic cells. In Jurkat cells, a decrease of MAL I-binding sites was observed as soon as $4 \mathrm{~h}$ after irradiation. In neutrophils, a strong decrease of MAL I-binding sites was only detectable in later apoptotic stages, when most of the cells were AxV positive but still PI negative. However, in both cases the loss of MAL I-binding sites preceded cell shrinkage.

Inhibition in viable cells of glycoprotein processing results in surface glycosylation similar to that of shrunken apoptotic cells. The glycoprotein-processing inhibitors castanospermine, 1-deoxymannojirimycin, and swainsonine were used to block protein glycosylation in the different processing pathways. During observation time the treatment with the processing inhibitors did not exert toxic effects on the cells (not shown). By analysing the glycosylation status of the plasma membrane of cells treated with the processing inhibitors (Figure 2) we found an increased binding of NPn and GSL II, and reduced binding of MAL I when compared with untreated cells. Interestingly, shrunken apoptotic yet PI impermeable cells expose additional NPn, GSL II, but less MAL I-binding sites on their surfaces as well. These data indicate that in shrunken late apoptotic cells sugar structures, which mainly comprise terminal sugar residues of immature intracellular proteins, get access to the plasma membrane.

After shrinkage apoptotic cells expose the ER-resident chaperon calnexin. Viable cells contain calnexin sequestered in their ER membranes and, therefore, no signal was detected for surface staining (Figure $3 a$ and $b$, wo). Calnexin could be detected by flow cytometry if antibodies against the protein got access to the intracellular compartment as it is the case for necrotic cells (Figure $3 a$ and $b$, intracellular). Lymphocytes contained less calnexin than neutrophils. Importantly, calnexin staining on the surfaces of $\mathrm{PI}$ impermeable cells could also be detected on late apoptotic cells after shrinkage (Figure $3 a$, spontaneous, population 3; Figure 3b, UV-B + STS). However, the amount of intracellular calnexin is significantly higher than that on apoptotic cells indicating that not the total ER pool of calnexin is reaching the cell surface.

The same observations were made using confocal microscopy (Figure $3 c$ and d). Viable cells did not show a surface signal (not shown). An intracellular staining of the entire cell was observed in necrotic and permeabilized cells while PI negative apoptotic cells exclusively displayed plasma membrane fluorescence. 
a

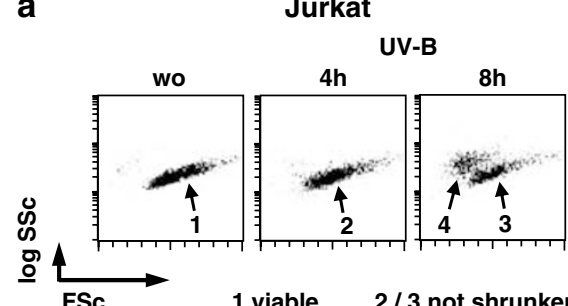

b

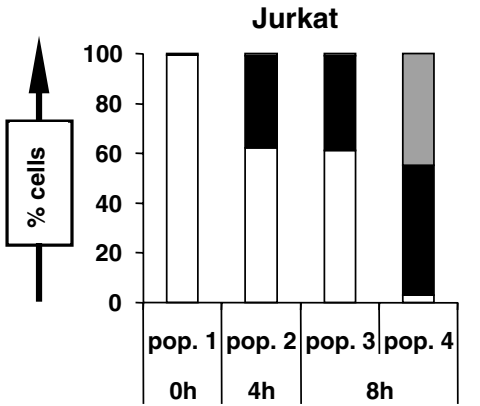

C

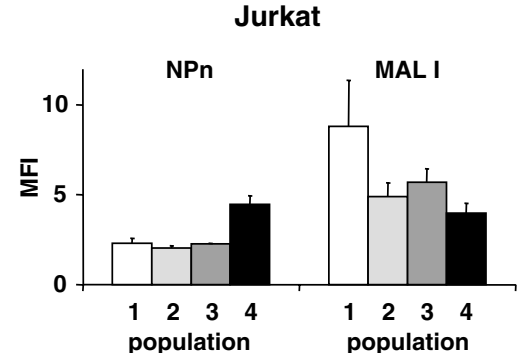

Neutrophils

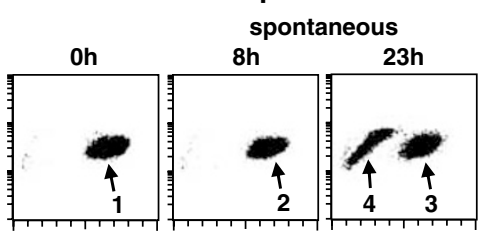

4 shrunken, late apoptotic

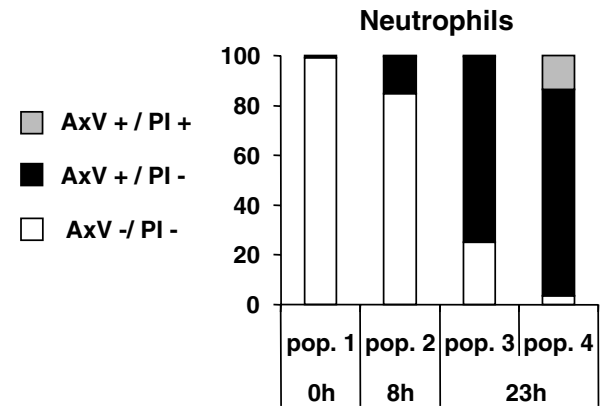

Neutrophils

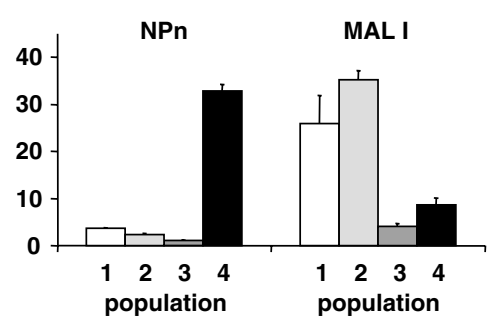

Figure 1 Analysis of lectin binding and AxV binding in presence of PI to Jurkat, and neutrophils by cytofluorometry. Treatments: untreated cells (wo), Jurkat cells apoptosis induction by irradiation with UV-B and culturing for up to $8 \mathrm{~h}$. Neutrophils underwent spontaneous apoptosis during $23 \mathrm{~h}$ of culture. (a) Changes of cellular morphology of Jurkat cells and neutrophils were analysed by FSc/SSc dot plots. Four populations were defined: viable (1), not shrunken, early apoptotic ( 2 and 3 ), and shrunken, late apoptotic cells (4). (b) Binding of $\mathrm{AxV}$ in the presence of $\mathrm{PI}$ to viable, and early as well as late apoptotic cells. (c) Binding of the lectins NPn and MAL I to viable, early apoptotic, and late apoptotic cells. Only cells, which excluded PI are shown. Shrunken, late apoptotic but still PI negative cells show increased binding of NPn in comparison to viable and to early apoptotic cells before shrinkage. Loss of MAL I-binding sites precedes shrinkage. Results are representative of three independent experiments. Samples were analysed in triplicates

Jurkat

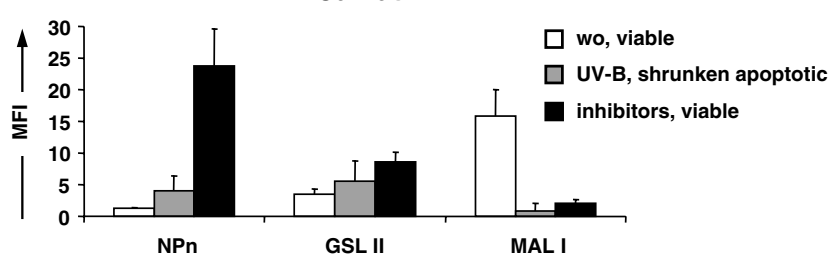

Figure 2 Analysis of lectin binding to Jurkat cells treated with glycoprotein processing inhibitors by cytofluorometry. Treatments: untreated cells (wo), Jurkat cells apoptosis induction by irradiation with UV-B and culturing for $8 \mathrm{~h}$ (UV-B), treatment with processing inhibitor cocktail for $24 \mathrm{~h}$ (inhibitors). NPn and GSL ॥ show increased binding to inhibitor treated cells and to shrunken apoptotic ones. Furthermore, MAL I binding is decreased in both cases. Results are representative of three independent experiments. Samples were analysed in triplicates

Calnexin and the UEA I ligands co-localized intracellular or at the plasma membrane of permeabilized and of apoptotic cells, respectively (Figure $3 \mathrm{~d}$ ). These data indicate that not only calnexin has translocated from the ER to the plasma membrane but, that also immature intracellular glycoproteins emerge at the plasma membranes of apoptotic cells.

PS exposure precedes lectin binding and calnexin exposure in the time course of apoptosis. Figure 4 shows a typical apoptotic time course of ageing neutrophils. Early during the progress of apoptosis, the cells expose PS at the outer surfaces of their plasma membranes as detected by AxV-staining with a continuous increase up to $85 \%$. The binding of the lectins GSL II and NPn, as well as that of calnexin increased continuously as well. The kinetics of the surface exposure of the lectinbinding sites and of calnexin were basically the same. Importantly, in the time course of apoptosis the exposure of PS clearly preceded both, the lectin binding and the calnexin exposure.

In apoptotic Ag8.H transfectants, a dysfunctional immunoglobulin $\mu$ chain gets access to the cell surface. Approximately half of all productively rearranged $\mu$ heavy chains $(\mathrm{HC})$ are unable to pair with surrogate light 
a

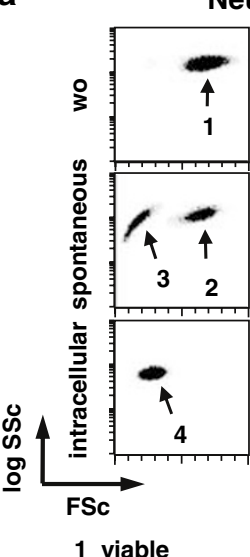

Neutrophils

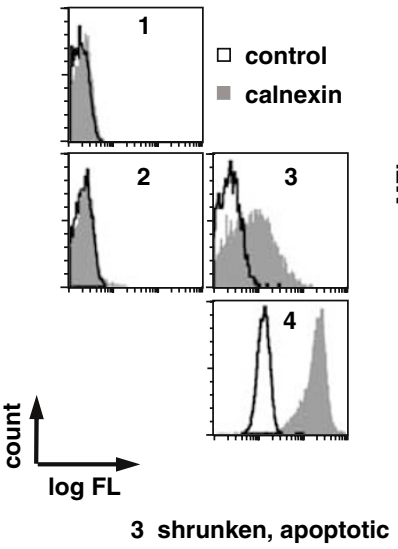

1 viable

not shrunken, apoptotic

shrunken, apoptotic b

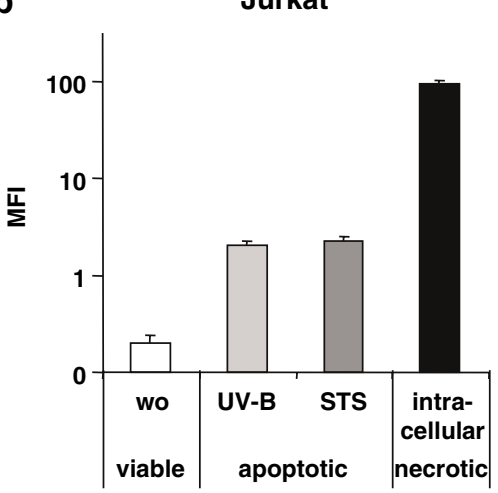

c

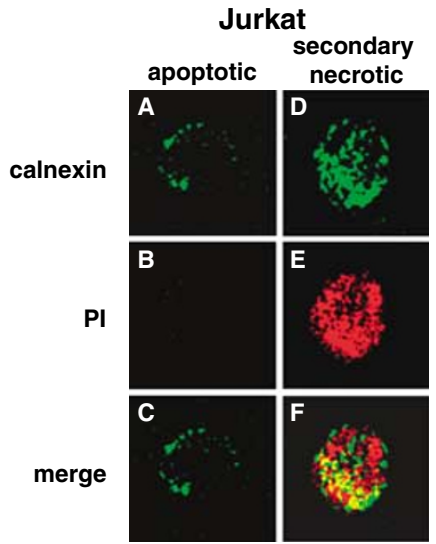

d

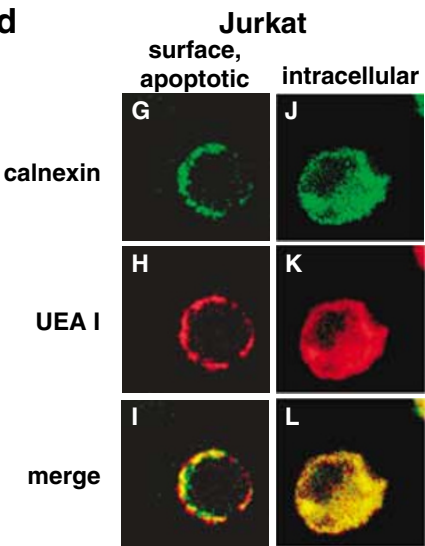

Figure 3 Analysis of calnexin exposure on neutrophils (a) and Jurkat cells (b-d). Neutrophils undergo spontaneous apoptosis during in vitro culture. Apoptosis of Jurkat cells was induced by irradiation (UV-B) or staurosporine (STS) and followed by 8 or $16 \mathrm{~h}$ of culture, respectively. Necrotic neutrophils and Jurkat cells were obtained by permeabilization of their plasma membranes (intracellular). (a) Four distinct populations can be defined by cytofluorometry: viable (1), not shrunken early apoptotic (2), shrunken late apoptotic (3), and necrotic cells (4). Binding of anti-calnexin (calnexin) is displayed in histograms. Anti-calnexin does not bind to the surface of viable and early apoptotic cells but to the plasma membrane of shrunken apoptotic cells. Necrotic cells bound markedly more anti-calnexin, when compared with apoptotic ones. (b) Binding of anti-calnexin to viable, apoptotic and necrotic Jurkat cells is displayed. Calnexin can not be detected on viable cells, but on shrunken apoptotic and on necrotic ones. (c) Binding of anti-calnexin to apoptozing Jurkat cells was analysed by confocal microscopy. Anti-calnexin (green) binds in a patchy pattern to the surface of apoptotic cells, which were impermeable for PI (red). (d) Binding of anti-calnexin and UEA I to apoptozing Jurkat cells was analysed by confocal microscopy. Calnexin epitopes and UEA I-(red) binding sites co-localize on the surfaces of apoptotic cells or intracellular. Results in (a) and (b) are representative of three independent experiments. Samples were analysed in quadruplicates. Results in (c) and (d) are representative of two independent experiments

chain and conventional immunoglobulin light chain. ${ }^{14}$ These dysfunctional $\mu \mathrm{HCs}$ are retained by the ER-resident heavy chain-binding protein $(\mathrm{BiP})$ and can, therefore, not reach the cell surface. ${ }^{15,16}$

To support our hypothesis that ER-resident components get access to the surface of late apoptotic cells, we used the Ag8. $\mathrm{H} \alpha$ cell line transfected with a nonpairing $\mu \mathrm{HC}(\mathrm{Ag} 8 . \mathrm{H} \alpha$ $\left.\mu_{\text {dys }}\right)$. This dysfunctional $\mu \mathrm{HC}$ is retained in the ER of viable cells and was exclusively detectable by intracellular staining as shown by flow cytofluorometry (Figure $5 \mathrm{a}$ ). By fluorescence microscopy we verified that vital Ag8. $\mathrm{H} \alpha \mu_{\text {dys }}$ cells did not show surface exposure of their $\mu \mathrm{HC}$ (Figure 5b, B). Furthermore, the morphological attributes are characteristic for vital cells (Figure $5 b, A$ ). In addition, viability of the cells was proven by the fact that their membranes did not allow the penetration of PI (Figure 5b, C). After treatment with UV-B and culture for a couple of hours, the dysfunctional $\mu \mathrm{HC}$ could be detected at the surface (Figure $5 b, E+H$ ) of apoptotic Ag8. $\mathrm{H} \alpha$ $\mu_{\text {dys }}$ cells, which show the typical high granularity and occasional blebs (Figure $5 b, D+G$ ). The membranes of those cells were still ion selective as shown by their impermeability for $\mathrm{PI}$ (Figure $5 \mathrm{~b}, \mathrm{~F}+\mathrm{I}$ ). In contrast, the membranes of necrotic Ag8. $\mathrm{H} \alpha \mu_{\text {dys }}$ cells were permeable for $\mathrm{PI}$ (Figure 5b, L). The antibodies against the $\mu \mathrm{HC}$ were able to enter necrotic cells and to bind the ER-resident $\mu \mathrm{HC}$ (Figure $5 b, K)$.

During apoptosis the lipophilic ER-Tracker ${ }^{\mathrm{TM}}$ Blue-White DPX and intracellular ganglioside GM1 are relocated to the plasma membrane of neutrophil granulocytes. The cell permeant, fluorescent lipid ER-Tracker ${ }^{\mathrm{TM}}$ Blue-White DPX specifically stains the ER of viable cells and can be 


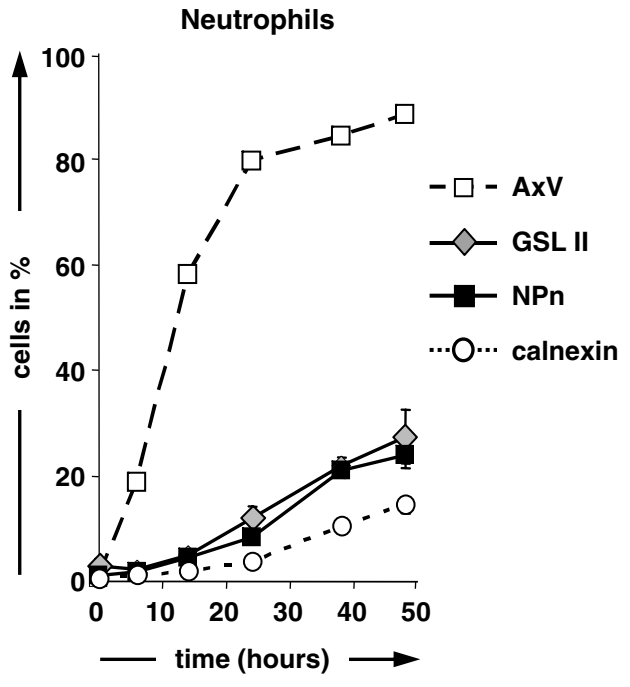

Figure 4 Time course of apoptosis for ageing neutrophils. Surface binding of GSL II, NPn, anti-calnexin (calnexin), and AxV to ageing neutrophils were analysed at various time points of culture. Time dependent increases of GSL II-, and NPn positive cells, as well as of cells binding anti-calnexin display similar kinetics but are delayed when compared to AxV binding. Results are representative of three independent experiments. Samples were analysed in quadruplicates

analysed by fluorescence microscopy (Figure 6a, C). The ER-Tracker penetrates intact cells and locates to the ER. We analysed the apoptosis of neutrophils that had been stained as freshly isolated viable cells. The uniform ER staining pattern, typical for viable cells (Figure 6a, C), drastically changed its appearance in apoptotic cells. The ER resident fluorescent lipid was now to be observed at the plasma membrane (Figure 6a, D).

When we compared the distribution of calnexin with that of the ER-Tracker, we found co-localization in viable as well as in apoptotic cells. In viable cells, both molecules were detected intracellular in the ER (Figure 6b, E-G). In apoptotic ones, both molecules partially located at the plasma membrane (Figure 6b, H-J).

We used cholera toxin $B(C T-B)$ to analyse the distribution of the glycolipid gangliosid (GM1) in viable and apoptotic neutrophils. In viable cells we detected GM1 at the plasma membrane and in intracellular stores. GM1 showed a uniform intracellular staining in viable cells while it appeared in a patchy pattern at the plasma membranes (Figure $6 c, K+N$ ). In apoptotic cells the GM1 staining pattern drastically changed. GM1 lost its uniform intracellular distribution and was found to cap in a distinct area of the plasma membrane (Figure $6 \mathrm{c}, \mathrm{Q}$ ). Comparing the location of $\mathrm{GM} 1$ and the ER-Tracker showed that both molecules co-localized inside viable cells and at the plasma membrane of apoptotic cells (Figure 6c, K-M, Q-S). Importantly, GM1 and the ER-Tracker did not co-localize at the plasma membrane of viable cells (Figure 6c, N-P).

Notably, each of those molecules, ER-Tracker, calnexin, and GM1, lost its uniform intracellular distribution when the cells entered the later phases of apoptosis. This suggests that these ER derived molecules translocate to the cell surface in shrunken apoptotic cells.

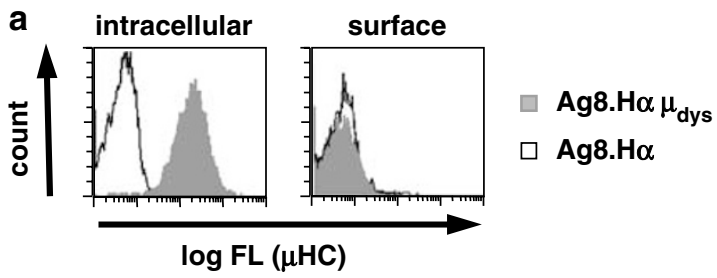

b Ag8.H $\alpha \mu_{\text {dys }}$
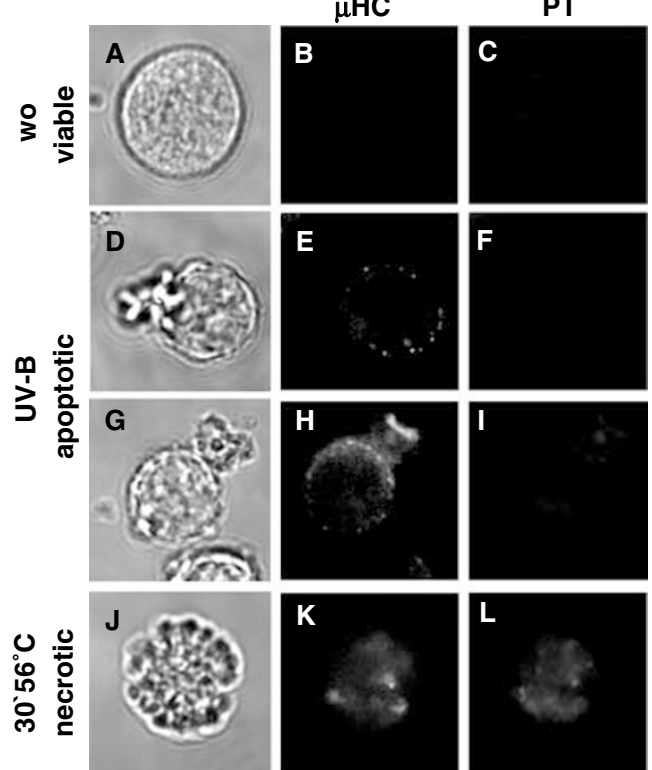

Figure 5 Cytofluorometric analysis (a) and fluorescence microscopic examination (b) of the expression of $\mu \mathrm{HC}$ in Ag8. $\mathrm{H} \alpha$ transfectants. The cells were treated as follows: untreated cells (wo), cells were induced to undergo apoptosis by irradiation with UV-B and then cultured for $10 \mathrm{~h}$ (UV-B). Necrosis was induced by incubating the cells for $30 \mathrm{~min}$ at $56^{\circ} \mathrm{C}\left(30^{\prime} 56^{\circ} \mathrm{C}\right)$ or by permeabilization of the cells (intracellular). (a) Surface and intracellular binding of anti- $\mu \mathrm{HC}$ to Ag8. $\mathrm{H} \alpha \mu_{\text {dys }}$ cells are displayed in histograms. The dysfunctional $\mu \mathrm{HC}$ can solely be detected intracellular in permabilized cells but not on the plasma membranes of viable cells. Staining of Ig-negative Ag8. $\mathrm{H} \alpha$ cells with anti- $\mu \mathrm{HC}$ served as control. (b) Binding of anti- $\mu \mathrm{HC}$ to viable, apoptotic, and necrotic $\mathrm{Ag} 8 . \mathrm{H} \alpha \mu_{\text {dys }}$ cells was analysed by fluorescence microscopy. Viable cells $(\mathrm{A}-\mathrm{C})$ show no binding of anti- $\mu \mathrm{HC}$. After apoptosis induction, $\mu \mathrm{HC}$ can be detected on the surfaces of dying $\mathrm{Ag} 8 . \mathrm{H} \alpha \mu_{\text {dys }}$ cells with an intact plasma membrane (D-F). In necrotic cells (G-I), anti- $\mu \mathrm{HC}$ mainly binds to intracellular targets. Results are representative of four independent experiments

Exposure of NPn-binding sites, calnexin, and GM1 on apoptozing neutrophils is abrogated when apoptotic membrane blebbing is blocked. The ROCK inhibitor Y-27632 was used to abrogate apoptotic cell blebbing of neutrophils undergoing spontaneous apoptosis. Figure 7a shows apoptotic neutrophils after $24 \mathrm{~h}$ of in vitro culture in the presence or absence of Y-27632. Mainly one population of not shrunken early and late apoptotic cells can be found in presence of the ROCK inhibitor. Staining with $\mathrm{AxV}$ and $\mathrm{PI}$ confirmed that the cells underwent apoptosis (Figure $7 \mathrm{~b}$ ). In Figure $7 \mathrm{c}$ we analysed the binding of NPn, anti-calnexin, and CT-B to apoptozing neutrophils treated with the ROCK inhibitor Y-27632 or not. Even if the cells were dead; in the presence of the bleb inhibitor no exposure of mannose 
a

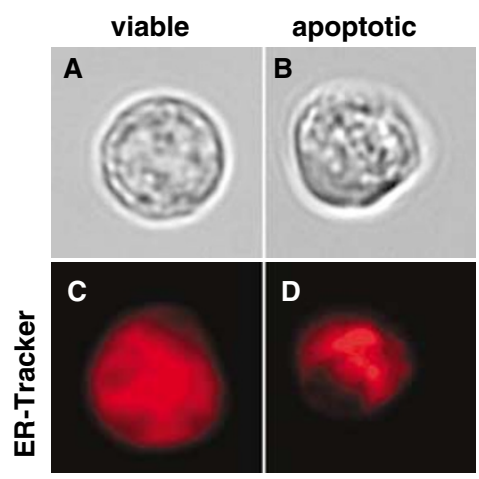

b
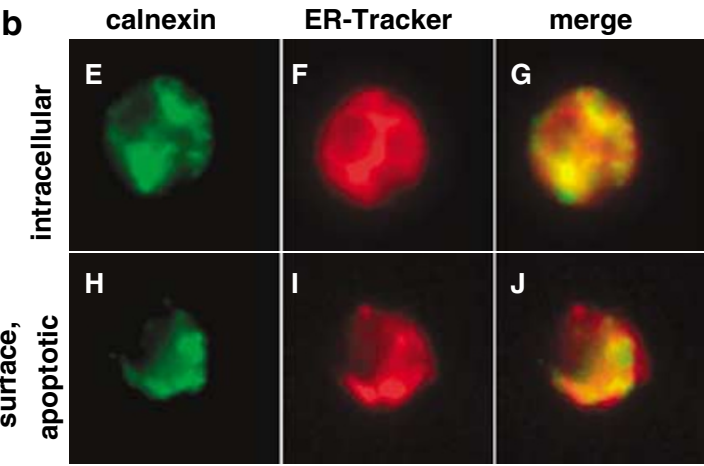

C
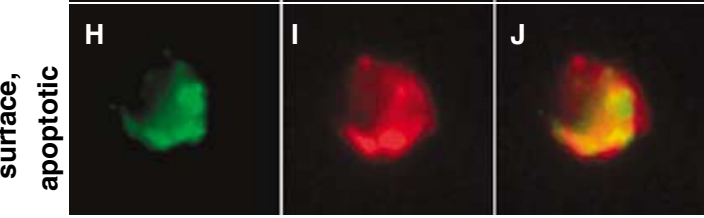

CT-B

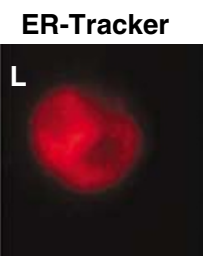

merge
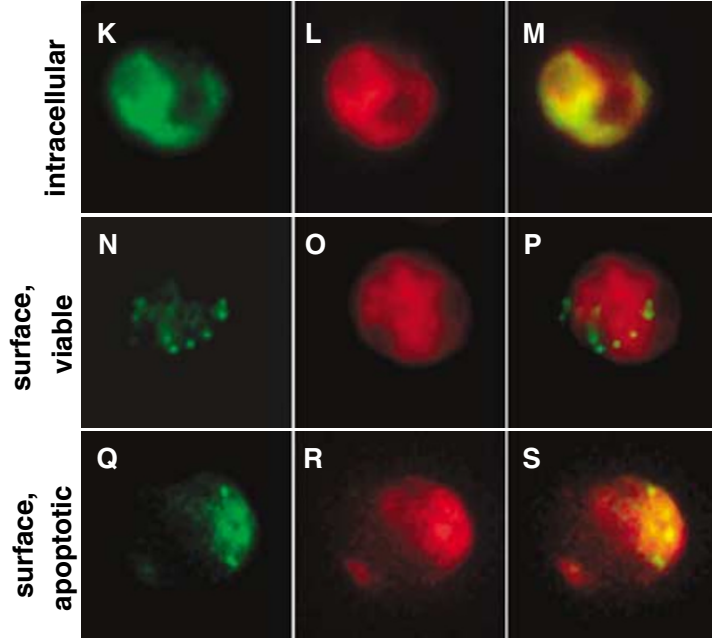

Figure 6 Fluorescence microscopic examination of neutrophils stained with the ER-Tracker, anti-calnexin, and CT-B. Viable neutrophils and neutrophils that underwent spontaneous apoptosis during an ageing process of $20 \mathrm{~h}$ were analysed. The ER-Tracker is shown in red; anti-calnexin and CT-B are displayed in green. (a) Viable and apoptotic neutrophils stained with the ER-Tracker are displayed. In viable neutrophils $(A+C)$, a uniform staining of the ER can be observed. In apoptotic neutrophils $(B+D)$, the dye has a different appearance - it is translocated toward the cell's surface. (b) Viable and apoptotic neutrophils double stained with anti-calnexin and the ER-Tracker are shown. Calnexin and ER-Tracker co-localize intracellular in permeabilized cells $(E-G)$ as well as on the surfaces of apoptotic neutrophils $(\mathrm{H}-\mathrm{J})$. Both dyes were not to be redetected on the surfaces of viable cells (not shown). (c) Viable and apoptotic neutrophils double stained with CT-B and the ER-Tracker are displayed. CT-B and ER-Tracker co-localized intracellular in permeabilized cells $(K-M)$, and on the surfaces of apoptotic cells $(Q-S)$. On the surfaces of viable neutrophils only CT-B can be detected in a patchy pattern $(\mathrm{N}+\mathrm{P})$. The ER-Tracker does not stain the surface of viable cells $(\mathrm{O}+\mathrm{P})$. Results are representative of two independent experiments containing epitopes, calnexin, and GM1 was observed. This is in striking contrast to cells not treated with Y-27632. The latter indicates once more that after shrinkage due to blebbing, apoptotic cells expose internal membrane-derived epitopes on their plasma membranes.

In late stages of apoptosis KDEL receptor transgenic HeLa cells expose ER membrane on their surfaces. To monitor the relocation of internal membranes to the cell surface during apoptotic membrane blebbing we used KDEL receptor-GFP transgenic HeLa cells. The KDEL receptor is a ER/cisGolgi compartment-located integral membrane protein. ${ }^{17}$ Apoptosis in KDEL receptor-GFP transgenic HeLa cells was induced by irradiation with UV-B. The distribution of the plasma membrane (red: Vybrant Dil dye) and the ER membrane (green: KDEL receptor-GFP) were monitored in the time laps (Figure 8 ). In viable cells ER membranes are located inside the cells. However, 240 min after UV-B, the plasma membrane starts blebbing and later on, ER membranes occupy the cells' surfaces. Interestingly, $350 \mathrm{~min}$ after irradiation the KDEL receptor positive membranes are also released via blebbing. These results clearly show that during apoptosis ER membrane is transported to the cells' surfaces to substitute plasma membrane that gets lost during the blebbing process.

\section{Discussion}

The exposure of PS on the outer surface of the plasma membrane is an early feature of apoptosis which coincides with structural changes of the plasma membrane ${ }^{7}$ and precedes cell shrinkage. PS, therefore, is detected by phagocytes as 'eat me' signal of apoptotic cells. If apoptotic cells escape immediate clearance, a second line of signals emerges which support clearance to avoid inflammation. $\mathrm{CRP}, \mathrm{C}_{1 \mathrm{q}}{ }^{18}$ and many other adaptor molecules like SP-A, SP$D$, and PTX-3 only bind to late apoptotic cells with a shrunken morphology. ${ }^{2}$ This shrinkage of apoptotic cells is at least partially due to the release of membrane coated blebs from their surfaces. ${ }^{8}$

The surface area of three dimensional bodies increases if divided into multiple smaller particles. This is also the case for blebbing apoptotic cells. As apoptotic cells do not lose their membrane integrity upon blebbing, new membranes have to be provided by the cells' internal stores; however, the exact pathway of this substitution remains to be clarified. We recently showed that GM1 was lost from the surfaces of aging neutrophils in early apoptosis and reappeared on the apoptotic cell surface after cell shrinkage. ${ }^{19}$ In apoptotic cells, it can be assumed that the early loss of the GM1 of ageing neutrophils is substituted from internal stores.

There is a close association of ER membranes with microtubules which, naturally, protrude to the plasma membrane $^{20}$ and a close association of ER membranes with the plasma membrane has recently been described. ${ }^{10}$ It has also been shown that the loss of surface membrane of phagocytes occurring during particle internalization is substituted by the ER. ${ }^{11}$ Therefore, ER membrane is a prime candidate for the supplementation of the plasma membrane. In this paper, we describe that the ER is supplying its membrane in favour of the 
a

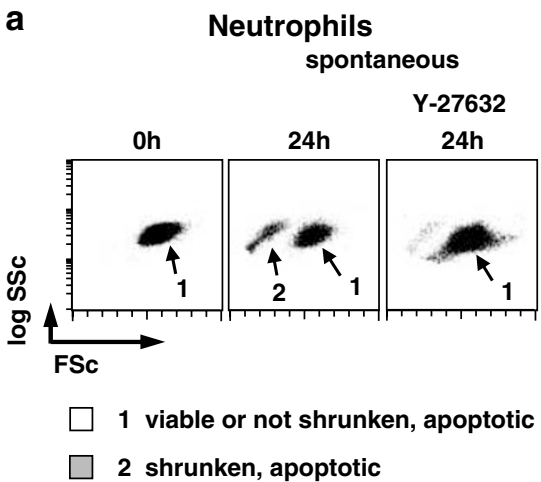

b

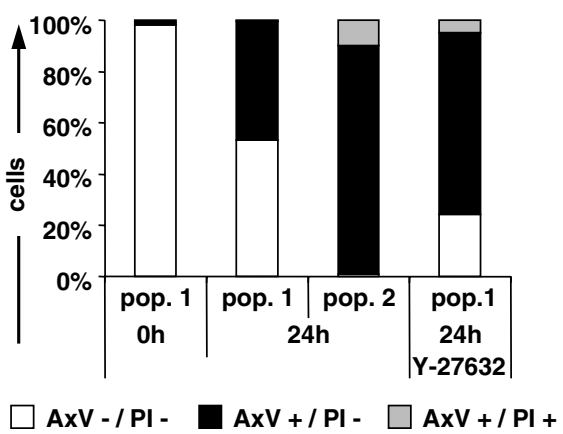

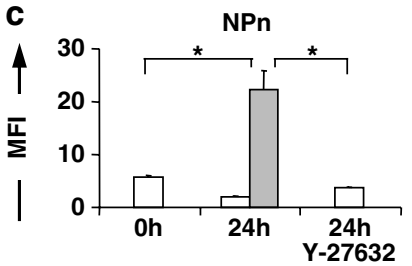
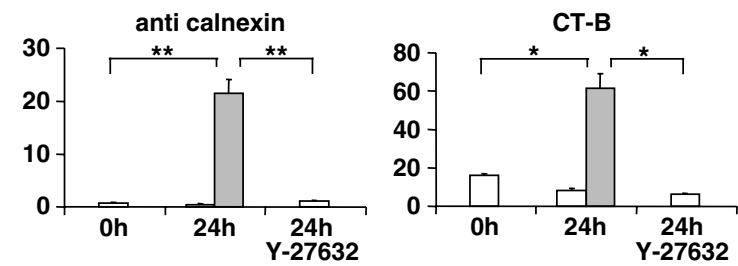

Figure 7 Analysis by cytofluorometry of NPn, anti-calnexin, and CT-B binding to apoptozing neutrophils treated with the ROCK inhibitor Y-27632. Neutrophils were analysed that underwent spontaneous apoptosis in the presence of the ROCK inhibitor Y-27632 during $24 \mathrm{~h}$ in vitro culture. (a) After $24 \mathrm{~h}$ ageing process two cell populations can be defined: not shrunken apoptotic cells (1), and shrunken apoptotic cells (2). In the presence of Y-27632 bleb formation is abrogated and, therefore, only one population was to be defined: not shrunken early and late apoptotic cells (1). (b) Staining with AxV/PI of viable, and shrunken as well as not shrunken apoptotic cells. (c) Binding to neutrophils undergoing apoptosis of NPn, anti-calnexin, and CT-B in the presence and absence of Y-27632. Late apoptotic cells, which had apoptozed in the presence of the blebbing inhibitor showed a similar binding of NPn, anti-calnexin, and CT-B as viable cells. Results are representative of two independent experiments. Samples were analyzed in triplicates. ${ }^{*} P<0.05 ;{ }^{* *} P<0.01$

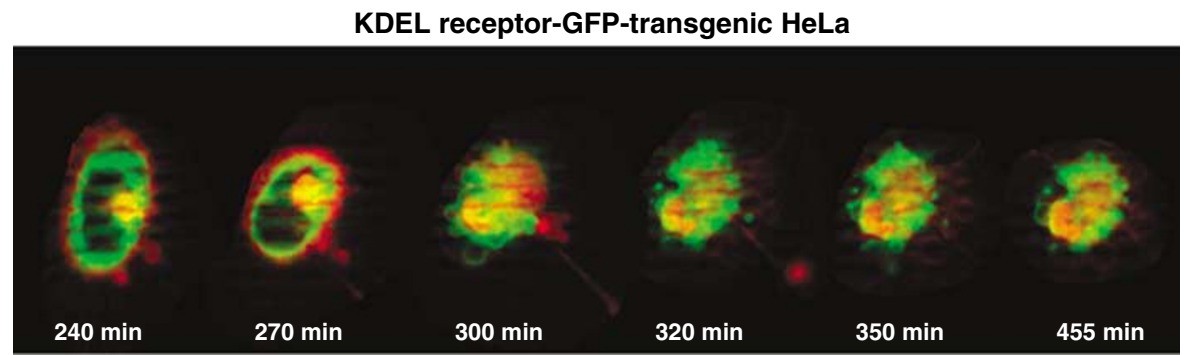

red: plasma membrane green: ER

Figure 8 Examination of apoptozing KDEL receptor-GFP-transgenic HeLa cells by fluorescence microscopy. In KDEL receptor-GFP-transgenic HeLa cells apoptosis was induced by irradiation with UV-B. The distribution of the plasma membrane (red) and the ER membrane (green) were monitored in the time laps as indicated. After 240 min plasma membrane starts blebbing followed by translocation of ER membranes to the cell's surface. Later on, also ER membrane derived blebs are released. Experiment was carried out in pentaplikates

plasma membrane. We observed that ER-resident proteins like calnexin, a dysfunctional $\mu \mathrm{HC}$, and the KDEL receptor were exposed at the surface of shrunken apoptotic cells. Calnexin is normally retained within the ER. However, a small fraction of calnexin is also expressed on the surface of various cells such as mastocytoma cells, murine splenocytes, fibroblast cells, and human HeLa cells. ${ }^{21}$ Therefore, we had selected cell lines and primary cells that did not expose calnexin if viable.

We also demonstrated that two lipids, GM1 and an ERTracker, which are solely localized in the ER membrane in early apoptotic cells, are redistributed to the plasma membrane in late apoptosis. In a recent study we have demonstrated that apoptotic cells show increased binding of the lectins GSL II, UEA I, and NPn. ${ }^{13}$ Importantly, exposure of PS and the loss of cellular volume preceded the increased lectin binding. Interestingly, GSL II, NPn, and, UEA I recognize sugar structures ( $N$-acetylglucosamine, polymannose, and fucose, respectively) predominantly found as terminal residues of immature glycoproteins during their processing in ER and Golgi. Here, we blocked the glycosylation of proteins using several processing inhibitors. Proteins that preserved their immature gylcosylation form were transported to the surfaces. Immature sugar structures could be detected by increased binding of NPn and GSL II. In nonapoptotic cells the majority of these sugars are located intracellulary. In late apoptotic stages they get exposed on the cell membrane suggesting that internal membranes containing incompletely 
processed proteins are translocated to the cells' surfaces under these conditions. We further underlined those results by the observation that additional GSL II-, UEA I-, and NPnbinding sites appear at the cells' surfaces with the same time course as calnexin, $\mu \mathrm{HC}_{\text {dys }}, \mathrm{GM} 1$ and the ER-Tracker.

MAL I specifically binds to carbohydrate chains containing sialic acid and particularly to the $\alpha \operatorname{Neu} 5 \mathrm{Ac}(2-3) \beta \mathrm{Gal}$ (1-4)GlcNAc/Glc sequence. ${ }^{22}$ MAL I has a high affinity for structures of mature glycoproteins. The terminal residues of oligosaccharides of mature glycoproteins are typically formed by sialic acid residues. Most commonly, they are displayed as a single terminal residue appended to an underlying galactose or GalNAc by a $\alpha-2.3-$ or $\alpha$-2.6-glycosidic linkage. We observed that the latter are lost from cell surfaces during apoptosis, reflected by a decreased binding of MAL I. This finding is consistent with the observation of Hart et al. ${ }^{23}$ They found reduced binding of the sialic acid-specific lectins derived from Limulus polyphemus Maackia amurensis (MAL II), and Sambucus nigra to surfaces of apoptotic neutrophils when compared with viable cells. However, they did not observe an increased binding of the galactose recognizing lectin peanut agglutinin implying that complete desialylation caused by activated sialidases ${ }^{24}$ did not represent a major carbohydrate modification event accompanying apoptosis. We observed that reduced binding of MAL I preceded cell shrinkage suggesting that very early membrane changes result in a partial loss of sialic acid. Later on, plasma membrane containing sialic acid glycosides get further lost during the blebbing process. Internal membranes containing immature glycosides replenish the membrane loss.

Our experiments with the blebbing inhibitor Y-27632 25 clearly showed that sugar structures, which mainly comprise terminal sugar residues of immature intracellular proteins, as well as calnexin and GM-1 get access to the plasma membrane only of shrunken apoptotic cells. The hypothesis of membrane exchange may help to explain how apoptotic cells rapidly alter their glycocalyx, simultaneously creating several potential clearance signals, without wasting much energy.

The surface changes, which we observed, were not only due to biochemical processes at the membrane but were also supplied by structures, which are sequestered inside viable cells. The recognition motifs were preformed internal structures of viable cells that merely get exposed after a period of membrane rearrangements during the late phases of apoptosis. This might be a result of the fact that ATP is limited in apoptotic cells and can, therefore, not be wasted for the energy consuming biosynthesis of new surface structures that serve as recognition signals. Furthermore, the preformed internal recognition epitopes of phagocytes may serve as highly economic 'eat-me' signals for late apoptotic and necrotic cells as well as for cellular debris. Future work will be to examine the phagocytosis of cells treated with glycoprotein processing inhibitors.

Apoptotic cell recognition can, theoretically, be based on several mechanisms. The recognition sites can (I) be synthesized de novo by a newly expressed enzymatic activity (like the induction of tissue transglutaminase and generation of lysophospholipids), can (II) be generated by (accidental) chemical modification (like oxidation of phospholipids), or can
(III) just be generated by the exposure of preformed epitopes which are usually sequestered inside viable cells (like PS). An apoptotic cell soon shuts down its power production and must, therefore, deal parsimoniously with its ATP reservoir. ${ }^{26}$ From this point of view, the exposure of preformed opsonin-binding sites that are sequestered inside viable cells is an economical method for the concomitant generation of several 'eat me' signals on surfaces of dying cells. We performed experiments with KDEL receptor-GFP transgenic HeLa cells to monitor the ER to plasma membrane trafficking during apoptosis. Importantly, after blebbing of the plasma membrane, ER membranes occupy the surfaces. After a while, those membranes were also released via blebbing. We conclude that during blebbing of apoptotic cells, the surface membrane loss is substituted by ER membranes containing immature glycoproteins and other structures that are not accessible on surfaces of viable cells.

\section{Materials and Methods}

Cells and culture conditions. Neutrophils were isolated from heparinized blood using density-gradient centrifugation (Ficoll-Histoprep/BAG, Lich, Germany). After centrifugation neutrophils were collected from the thin cell layer immediately above the erythrocytes. Residual erythrocytes were removed by hypotonic lysis. The hematopoetic (lymphatic) cell line Jurkat cells and HeLa cells were obtained from American Type Culture Collection (ATCC).

Neutrophils, and Jurkat cells were cultured at $37^{\circ} \mathrm{C}$ in a humidified atmosphere in the presence of $5.5 \% \mathrm{CO}_{2}$ in culture medium (RPMI 1640) supplemented with $10 \%$ heat inactivated foetal calf serum (FCS), $1 \%$ glutamine, 1\% HEPES, and $1 \%$ penicillin-streptomycin (all from Gibco, Eggenstein, Germany). Ag8.H, a subclone of the Ig-negative mouse plasmocytoma cell line Ag8.653, ${ }^{27}$ stable Ag8.H transfectants and $\mathrm{KDEL}$ receptor transgenic HeLa cells were maintained at $37^{\circ} \mathrm{C}$ and $5 \% \mathrm{CO}_{2}$ in complete RPMI (RPMI 1640 supplemented with $50 \mathrm{U} / \mathrm{ml}$ penicillin, $50 \mu \mathrm{g} / \mathrm{ml}$ streptomycin, $5 \% \mathrm{FCS}, 1 \mathrm{mM}$ sodium pyruvate and $2 \mathrm{mM}$ L-glutamine; all from Invitrogen, Karlsruhe, Germany).

Plasmid construction and stable transfections. To construct an expression vector encoding murine $\lg \alpha$, which is required for surface transport of immunoglobulin receptor complexes ${ }^{28}$ a cDNA fragment was amplified by PCR with an appropriate forward (TTGGATCCACGATGCCAGGGGGTCTAGA, containing a BamHI restriction site) and backward primer (TTGAATTCCAGTCATGGCTT TTCCAGCT, containing an EcoRI restriction site) from the murine lymphoma B-cell line $\mathrm{CH} 27$ and cloned into the BamHI and EcoRI site of the mammalian expression vector pEF1/myc-His (Invitrogen, Karlsruhe, Germany). Ag8.H cells were electroporated with the $\lg \alpha$-encoding plasmid as described $\left(250 \mathrm{~V}, 960 \mu \mathrm{F}^{29}\right)$. Stable clones were selected in complete medium supplemented with $1 \mathrm{mg} / \mathrm{ml} \mathrm{G} 418$ (PAA, Pasching, Germany). The Ig $\alpha$-positive clone Ag8.H $\alpha$ was transfected by electroporation with the expression vector $p \mu$ dys.gpt encoding the IgL-nonpairing, dysfunctional $\mu \mathrm{HC}$ carrying the VH81XF segment $\left(\mu_{\text {dys }}{ }^{15,29}\right)$. Stable transfectants were selected in complete RPMI supplemented with $1 \mathrm{mg} / \mathrm{ml} \mathrm{G} 418,1.25 \mu \mathrm{g} / \mathrm{ml}$ mycophenolic acid, $250 \mu \mathrm{g} / \mathrm{ml}$ xanthine, $100 \mu \mathrm{M}$ hypoxanthine and $16 \mu \mathrm{M}$ thymidine (all from Sigma Aldrich, Taufkirchen, Germany). Single cell clones were established by limiting dilution and analysed by flow cytometry.

Human Erd2 (KDEL receptor) CDNA in PECFP-N1 ${ }^{30}$ was a kind gift of Margarita Cabrera. The plasmid pECFP-N1-Erd2 was cleaved with Agel and Notl (New England Biolabs, MA, USA) and cloned into corresponing sites of pEGFP-N1 (Clontech, CA, USA). The sequence integrity of the clone was verified by DNA sequencing. The cells were transfected in 24-well culture plates using LipofectAMINE 2000 (Invitrogen, Karlsruhe) in OPTI-MEM following the manufacturer's instructions. Transfection efficiency and expression of the GFP-Erd2 fusion protein was confirmed through fluorescence microscopy. Stable transfected cells were obtained by clonal selection in culture medium supplemeted with $1 \mathrm{mg} / \mathrm{ml}$ G418 (Roth, Karlsruhe).

Reagents and antibodies. Fluorescein isothiocyanate- (FITC) labelled recombinant human $\mathrm{AxV}$ was purchased from responsif $\mathrm{GmbH}$ (Erlangen, Germany), FITC-labelled cholera toxin subunit B (CT-B) and PI from Sigma 
(Munich, Germany), the ER-Tracker ${ }^{\mathrm{TM}}$ Blue-White DPX and the Vybrant ${ }^{\mathrm{TM}}$ Dil cell labelling solution from Invitrogen (Karlsruhe, Germany), and the Cytofix/CytoPerm Plus ${ }^{\mathrm{TM}}$ Kit as well as the FITC-labelled monoclonal antibody against calnexin from Pharmingen (Heidelberg, Germany). Unconjugated lectin Narcissus pseudonarcissus (NPn), FITC-labelled lectin Griffonia simplificolia II (GSL II), Rhodamine-labelled and FITC-labelled lectin Ulex europaeus I (UEA I), and FITClabelled lectin Maackia amurensis I (MAL I) were purchased from Vector Laboratories (distributed by Linaris, Wertheim, Germany). NPn was labelled by using the FluoroTag fluorescein isothiocyanate (FITC) conjugation kit (Sigma, Munich, Germany). The ROCK inhibitor Y-27632 as well as the processing inhibitors castanospermine, 1-deoxymannojirimycin, and swainsonine were purchased from Calbiochem (Darmstadt, Germany). Affinity-purified goat antibodies directed against the $\mathrm{HC}$ of $\mathrm{IgM}$ were purchased from Southern Biotechnology (Birmingham, AL, USA), antibodies against mouse $\mathrm{FC} \gamma$ from Jackson ImmunoResearch Laboratories (distributed by Dianova, Hamburg, Germany), and mouse lgG1, $\kappa$ (from MOPC21 myeloma cell line) from Sigma Aldrich (Taufkirchen, Germany). Affinity-purified goat antibodies against mouse $\alpha \mathrm{H}$-chain were labelled with the Cy5 labelling kit from Amersham Biosciences (Freiburg, Germany). The monoclonal mouse $\operatorname{lgG} 1, \kappa$ antibody $24 \mathrm{C} 2.5$ against the intracellular tail of mouse $\lg \alpha$ was described previously. ${ }^{31}$

Induction and detection of apoptosis and necrosis. Apoptosis in Jurkat cells, and Ag8.H $\alpha$ transfectants were induced by irradiation with UVB $\left(180 \mathrm{~mJ} / \mathrm{cm}^{2}\right)$ or treatment with $0.5 \mu \mathrm{M}$ staurosporine in culture medium. After apoptosis induction the cells were cultured under standard conditions. Neutrophils underwent spontaneous progressive apoptosis during the ageing process. Primary necrosis was induced by incubation of the cells at $56^{\circ} \mathrm{C}$ for $30 \mathrm{~min}$.

Monitoring of apoptosis and necrosis was performed by microscopic, and by flow cytofluorometry employing the following methods: (I) AxV staining in the presence of $\mathrm{PI}$; and (II) morphological changes of the cells (FSc versus SSc).

Inhibition assays. The ROCK inhibitor $\mathrm{Y}-27632$ was used at $50 \mu \mathrm{M}$ to abrogate apoptotic cell blebbing of neutrophils undergoing spontaneous apoptosis during $24 \mathrm{~h}$ of in vitro culture.

Castanospermine, 1-deoxymannojirimycin, and swainsonine are inhibitors of glycoprotein processing. Jurkat cells were cultured in $\mathrm{R}^{10}$ containing an inhibitor cocktail $(50 \mu \mathrm{g} / \mathrm{ml}$ castanospermine, $100 \mu \mathrm{g} / \mathrm{ml}$ 1-Deoxymannojirimycin, $1 \mu \mathrm{g} / \mathrm{ml}$ swainsonine) for $24 \mathrm{~h}$.

Flow cytofluorometry and microscopy analysis. To detect surface exposure of altered carbohydrates, the cells were stained with the following fluorescence-labelled lectins: (I) GSL II-FITC ( $\alpha$ - or $\beta$-linked N-acetylglucosamine at the nonreducing terminal of oligosaccharides), (II) NPn-FITC (polymannose structures containing $(\alpha-1,6)$ linkages or galactomannans), (III) UEA I-FITC or Rhodamine ( $\alpha$-linked fucose residues), (IV) MAL I-FITC (sialic acid in the trisaccharide sequence $\alpha \operatorname{Neu} 5 \mathrm{Ac}(2-3) \beta \mathrm{Gal}(1-4) \mathrm{GlcNAc} / \mathrm{Glc})$. MAL I had to be used in a 25 times lower concentration than described previously ${ }^{13}$ since the undiluted lectin was severely affecting shape and viability of the cells. The MAL Idilution we used in the experiments of this publication was not toxic during the 30 min incubation period. The surface expression of calnexin was detected using an antibody against an ER luminal domain of calnexin. To follow the exposure of GM1 the cells were stained with FITC-labelled CT-B. The surface staining of the cells was performed as follows: $100 \mu$ lof the cell suspension $\left(1 \times 10^{5}\right.$ cells) were incubated at $4^{\circ} \mathrm{C}$ with $4 \mu \mathrm{g}$ of the labelled lectin ( $0.16 \mu \mathrm{g} \mathrm{MAL} \mathrm{I),} 2 \mu \mathrm{g}$ anti-calnexin, or $0.2 \mu \mathrm{g} \mathrm{CT}$ B. To detect necrotic cells $0.5 \mu \mathrm{g} \mathrm{PI}$ was added to all samples. After $30 \mathrm{~min}$ the cells were washed with PBS and then fixed with PBS containing $1 \%$ (w/v) PFA. For intracellular stainings the cells were permeabilized using the Cytofix/CytoPerm Plus $^{\mathrm{TM}}$ Kit according to the manufacturer's instruction and then stained as mentioned. For membrane staining of the Ag8. H transfectants $5 \times 10^{5}$ cells were incubated in PBS supplemented with $2 \%$ FCS, $0.1 \% \mathrm{NaN}_{3}$ (FACS-PBS) and appropriate antibodies for $30 \mathrm{~min}$ on ice. For cytoplasmic staining, $5 \times 10^{5}$ cells were first fixed in $4 \%$ paraformaldehyde dissolved in PBS at RT for $10 \mathrm{~min}$ permeabilized with $0.1 \%$ Tween 20 at $37^{\circ} \mathrm{C}$ for $15 \mathrm{~min}$ and stained at RT for $15 \mathrm{~min}$ with appropriate antibodies. To analyse the distribution of the ER in neutrophils we used the ER-Tracker ${ }^{\text {TM }}$ Blue-White DPX. KDEL receptor transgenic HeLa cells were labelled using the Vybrant ${ }^{\mathrm{TM}}$ Dil cell labelling solution.

Flow cytofluorometry was performed with a FACSCalibur (BD Biosciences, CA USA), or with an EPICS ${ }^{\text {TM }}$ cytofluorometer (Coulter, Hialeah, USA). Electronic compensation was used to eliminate bleed through of fluorescence. Data analysis was performed with the CellQuest Pro program (v4.0.2) or with Coulter $\mathrm{XL}^{\mathrm{TM}}$ software (v3), respectively.

Fluorescence microscopy was performed with an IX70 inverted fluorescence microscope (Olympus, Hamburg, Germany). Confocal microscopy was performed with a Leica DMR confocal microscope (Leica, Wetzlar, Germany), software version 2.00

Image aquisition of apoptozing KDEL receptor-GFP-transgenic HeLa cells was carried out with a Zeiss AxioCam MRm digital camera attached to a Zeiss Axiovert $200 \mathrm{M}$ inverted microscope. The microscope was equipped with a Zeiss ApoTome and an incubator Zeiss XL-3 for life microscopy. Image acquisition was performed under $5 \% \mathrm{CO}_{2}$ at $37^{\circ} \mathrm{C}$ in a humidified atmosphere for $16 \mathrm{~h}$. Images of full sensor resolution (1.3MP) were captured every $5 \mathrm{~min}$ until the end of experiment using a $\times 40$ objective (Zeiss Plan-Neofluar $\times 40 / 0,75$ ) and the following filter sets for fluorescence (all from Zeiss): Red: Filter set 20 (exc.: BP 546/12; emm. 575-640); Green: Filter set 38 (exc.: BP 470/40; emm. 525/50).

Acknowledgements. We thank Dirk Mielenz for reagents and help with confocal microscopy, Mirjam Metzner for establishing stable transfectants, Margarita Cabrera for providing Human Erd2 (KDEL receptor) cDNA, and Roman Gerlach for help with Zeiss Axiovert $200 \mathrm{M}$ inverted microscope. This work was supported by the Interdisciplinary Center for Clinical Research (IZKF) (project number A4) at the University Hospital of the University of Erlangen-Nuremberg, by 'Deutsche Forschungsgemeinschaft' SFB 643 (project B5), by the European Commissions [EU (QLK3-CT-2002-02017_APOCLEAR)], and in part by project grant SFB 466 and research grant JA 968/2 to H-MJ from the Deutsche Forschungsgemeinschaft (DFG). SF and KH were supported by the DFG research training grant GK592 and the University of Erlangen's ELAN program.

1. Fadok VA, Xue D, Henson $P$. If phosphatidylserine is the death knell, a new phosphatidylserine-specific receptor is the bellringer. Cell Death Differ 2001; 8: 582-587.

2. Hart SP, Smith JR, Dransfield I. Phagocytosis of opsonized apoptotic cells: roles for 'old-fashioned' receptors for antibody and complement. Clin Exp Immunol 2004; 135: 181-185.

3. Voll RE, Herrmann M, Roth EA, Stach C, Kalden JR, Girkontaite I. Immunosuppressive effects of apoptotic cells. Nature 1997; 390: 350-351.

4. Kothakota S, Azuma T, Reinhard C, Klippel A, Tang J, Chu K et al. Caspase-3-generated fragment of gelsolin: effector of morphological change in apoptosis. Science 1997; 278: 294-298.

5. Kondo T, Takeuchi K, Doi Y, Yonemura S, Nagata S, Tsukita S. ERM (ezrin/radixin/ moesin)-based molecular mechanism of microvillar breakdown at an early stage of apoptosis. J Cell Biol 1997; 139: 749-758.

6. Heyder P, Gaipl US, Beyer TD, Voll RE, Kern PM, Stach C et al. Early detection of apoptosis by staining of acid-treated apoptotic cells with FITC-labeled lectin from Narcissus pseudonarcissus. Cytometry 2003; 55A: 86-93.

7. Appelt U, Sheriff A, Gaipl US, Kalden JR, Voll RE, Herrmann M. Viable, apoptotic and necrotic monocytes expose phosphatidylserine: cooperative binding of the ligand Annexin $\mathrm{V}$ to dying but not viable cells and implications for PS-dependent clearance. Cell Death Differ 2005; 12: 194-196.

8. Coleman ML, Sahai EA, Yeo M, Bosch M, Dewar A, Olson MF. Membrane blebbing during apoptosis results from caspase-mediated activation of ROCK I. Nat Cell Biol 2001; 3: 339345.

9. Morris RG, Hargreaves AD, Duvall E, Wyllie AH. Hormone-induced cell death. 2. Surface changes in thymocytes undergoing apoptosis. Am J Pathol 1984; 115: 426-436.

10. Latz E, Schoenemeyer A, Visintin A, Fitzgerald KA, Monks BG, Knetter CF et al. TLR9 signals after translocating from the ER to CpG DNA in the lysosome. Nat Immunol 2004; 5 : 190-198.

11. Gagnon E, Duclos S, Rondeau C, Chevet E, Cameron PH, Steele-Mortimer O et al. Endoplasmic reticulum-mediated phagocytosis is a mechanism of entry into macrophages. Cell 2002; 110: 119-131.

12. Bilyy RO, Antonyuk VO, Stoika RS. Cytochemical study of role of alpha-D-mannose- and beta-D-galactose-containing glycoproteins in apoptosis. J Mol Histol 2004; 35: 829-838.

13. Franz S, Frey B, Sheriff A, Gaipl US, Beer A, Voll RE et al. Lectins detect changes of the glycosylation status of plasma membrane constituents during late apoptosis. Cytometry $A$ 2006; 23: 23

14. ten Boekel $E$, Melchers F, Rolink AG. Changes in the $V(H)$ gene repertoire of developing precursor $B$ lymphocytes in mouse bone marrow mediated by the pre- $B$ cell receptor. Immunity 1997; 7: 357-368.

15. Kline GH, Hartwell L, Beck-Engeser GB, Keyna U, Zaharevitz S, Klinman NR et al. Pre-B cell receptor-mediated selection of pre-B cells synthesizing functional mu heavy chains. J Immunol 1998; 161: 1608-1618.

16. Haas IG, Wabl M. Immunoglobulin heavy chain binding protein. Nature 1983; 306 : 387-389. 
17. Cabrera M, Muniz M, Hidalgo J, Vega L, Martin ME, Velasco A. The retrieval function of the KDEL receptor requires PKA phosphorylation of its C-terminus. Mol Biol Cell 2003; 14: 4114-4125.

18. Gaipl US, Kuenkele S, Voll RE, Beyer TD, Kolowos W, Heyder P et al. Complement binding is an early feature of necrotic and a rather late event during apoptotic cell death. Cell Death Differ 2001; 8: 327-334.

19. Sheriff A, Gaipl US, Franz S, Heyder P, Voll RE, Kalden JR et al. Loss of GM1 surface expression precedes annexin V-phycoerythrin binding of neutrophils undergoing spontaneous apoptosis during in vitro aging. Cytometry A 2004; 62: 75-80.

20. Terasaki M, Chen LB, Fujiwara K. Microtubules and the endoplasmic reticulum are highly interdependent structures. J Cell Biol 1986; 103: 1557-1568.

21. Okazaki Y, Ohno H, Takase K, Ochiai T, Saito T. Cell surface expression of calnexin, a molecular chaperone in the endoplasmic reticulum. J Biol Chem 2000; 275: 35751-35758.

22. Imberty A, Gautier C, Lescar J, Perez S, Wyns L, Loris R. An unusual carbohydrate binding site revealed by the structures of two Maackia amurensis lectins complexed with sialic acid-containing oligosaccharides. J Biol Chem 2000; 275: 1754117548.

23. Hart SP, Ross JA, Ross K, Haslett C, Dransfield I. Molecular characterization of the surface of apoptotic neutrophils: implications for functional downregulation and recognition by phagocytes. Cell Death Differ 2000; 7: 493-503.
24. Cross AS, Wright DG. Mobilization of sialidase from intracellular stores to the surface of human neutrophils and its role in stimulated adhesion responses of these cells. J Clin Invest 1991; 88: 2067-2076.

25. Sebbagh M, Renvoize C, Hamelin J, Riche N, Bertoglio J, Breard J. Caspase-3-mediated cleavage of ROCK I induces MLC phosphorylation and apoptotic membrane blebbing Nat Cell Biol 2001; 3: 346-352.

26. Chiarugi $A$. Simple but not simpler: toward a unified picture of energy requirements in cell death. FASEB J 2005; 19: 1783-1788.

27. Kearney JF, Radbruch A, Liesegang B, Rajewsky K. A new mouse myeloma cell line that has lost immunoglobulin expression but permits the construction of antibody-secreting hybrid cell lines. J Immunol 1979; 123: 1548-1550.

28. Hombach J, Leclercq L, Radbruch A, Rajewsky K, Reth M. A novel 34-kd protein co-isolated with the IgM molecule in surface IgM-expressing cells. EMBO J 1988; 7: 3451-3456.

29. Keyna U, Beck-Engeser GB, Jongstra J, Applequist SE, Jack HM. Surrogate light chaindependent selection of Ig heavy chain V regions. J Immunol 1995; 155: 5536-5542.

30. Lewis MJ, Pelham HR. A human homologue of the yeast HDEL receptor. Nature 1990; 348: 162-163.

31. Mielenz D, Ruschel A, Vettermann C, Jack HM. Immunoglobulin mu heavy chains do not mediate tyrosine phosphorylation of Ig alpha from the ER-cis-Golgi. J Immunol 2003; 171 3091-3101. 